\title{
Abstract
}

\section{Dynamic Modeling of the SSDI Application Timing Decision: The Importance of Policy Variables}

\author{
Richard V. Burkhauser, J. S. Butler and Gulcin Gumus
}

This paper analyzes the importance of policy variables in the context of Social Security Disability Insurance (SSDI) application timing decision. Previously, we explicitly modeled the optimal timing of SSDI application using dynamic structural models. We estimated these models using data from the Health and Retirement Study (HRS). This paper uses option value model estimates to simulate application timing under alternative SSDI policy formulations. We consider changes in three policy variables: benefit levels, acceptance rates, and employer accommodation. Our simulations suggest all these changes would have substantial effects on expected spell lengths until application and on lifetime application rates, and hence on SSDI caseloads.

Published in: (IZA), 2003, Discussion Paper No. 942.

For full text see publisher's website:

ftp://repec.iza.org/RePEc/Discussionpaper/dp942.pdf 